\title{
Action of methotrexate and tofacitinib on directly stimulated and bystander-activated lymphocytes
}

\author{
ELISA PISCIANZ ${ }^{1}$, VANESSA CANDILERA ${ }^{2}$, ERICA VALENCIC ${ }^{1}$, CLAUDIA LOGANES $^{2}$, \\ GRETA PARON $^{2}$, SARA DE IUDICIBUS ${ }^{1}$, GIULIANA DECORTI ${ }^{3}$ and ALBERTO TOMMASINI ${ }^{4}$ \\ ${ }^{1}$ Department of Advanced Diagnostic and Clinical Trials, Institute for Maternal and Child Health, \\ IRCCS 'Burlo Garofolo', I-34137 Trieste; Departments of ${ }^{2}$ Medical, Surgical and Health Sciences and ${ }^{3}$ Life Sciences, \\ University of Trieste, I-34100 Trieste; ${ }^{4}$ Department of Paediatrics, Institute for Maternal and Child Health, \\ IRCCS ‘Burlo Garofolo’, I-34137 Trieste, Italy
}

Received November 25, 2015; Accepted March 7, 2016

DOI: $10.3892 / \mathrm{mmr} .2016 .5263$

\begin{abstract}
Chronic inflammation associated with autoimmune activation is characteristic of rheumatic diseases from childhood to adulthood. In recent decades, significant improvements in the treatment of these types of disease have been achieved using disease modifying anti-rheumatic drugs (DMARDs), such as methotrexate (MTX) and, more recently, using biologic inhibitors. The recent introduction of kinase inhibitors (for example, tofacitinib; Tofa) further increases the available ARDs. However, there are patients that do not respond to any treatment strategies, for whom combination therapies are proposed. The data regarding the combined action of different drugs is lacking and the knowledge of the mechanisms of ARDs and their actions upon pathogenic lymphocytes, which are hypothesized to sustain disease, is poor. An in vitro model of inflammation was developed in the current study, in which stimulated and unstimulated lymphocytes were cultured together, but tracked separately, to investigate the action of MTX and Tofa on the two populations. By analysing lymphocyte proliferation and activation, and cytokine secretion in the culture supernatants, it was established that, due to the presence of activated cells, unstimulated cells underwent a bystander activation that was modulated by the ARDs. Additionally, varying administration schedules were demonstrated to affect lymphocytes differently in vitro,
\end{abstract}

Correspondence to: Dr Elisa Piscianz, Department of Advanced Diagnostic and Clinical Trials, Institute for Maternal and Child Health, IRCCS 'Burlo Garofolo', Via dell'Istria 65/1, I-34137 Trieste, Italy

E-mail: elisa.piscianz@gmail.com

Abbreviations: MTX, methotrexate; Tofa, tofacitinib; DMARDs, disease modifying anti-rheumatic drugs; PBMCs, peripheral blood mononuclear cells

Key words: anti-rheumatic drugs, tofacitinib, methotrexate, bystander effect, pathogenic lymphocytes either directly or via bystander activation. Furthermore, MTX and Tofa exerted different effects; while MTX showed an antiproliferative effect, Tofa marginally effected activation, although only a slight antiproliferative action, which could be potentiated by sequential treatment with MTX. Thus, it was hypothesized that these differences may be exploited in sequential therapeutic strategies, to maximize the anti-rheumatic effect. These findings are notable and must be accounted for, as bystander-activated cells in vivo could contribute to the spread of autoimmune activation and disease progression.

\section{Introduction}

Rheumatic diseases are characterized by inflammation and autoimmune activation. This is more evident in paediatric patients, where the pathogenesis of rheumatic diseases appears to possess intermediate features between two ends of an immunologic disease continuum, represented by monogenic autoinflammatory and autoimmune diseases (1).

This complex pathogenesis may account for the difficulties in completely curing rheumatic diseases, regardless of the improved therapeutic approaches, which relieve symptoms and prevent severe complications in the majority of cases. In addition, the requirement for enduring treatments may have a negative impact on patient quality of life and upon health costs (2).

Current treatments are based on glucocorticoids, disease modifying anti-rheumatic drugs (DMARDs) [such as methotrexate (MTX) and leflunomide] and on biological drugs targeting inflammatory cytokines [anti-interleukin (IL)-1, anti-IL-6, anti-tumour necrosis factor (TNF)- $\alpha$ ] or targeting immune cell activation (such as CTLA4Ig). Furthermore, novel molecules, such as kinase inhibitors [for example, tofacitinib (Tofa) or baricitinib] have recently been introduced (3-6).

Although the number of available drugs is increasing, treatment options and schedules remain largely empirical, and are based on their ability to delay or prevent tissue damage in clinical trials. Considering the role of inflammation and autoimmunity in rheumatic disorders, approaches to treatment include drugs with prevalent anti-inflammatory action (such as glucocorticoids and anti-cytokine biologics) and immunosuppressive agents (such as kinase inhibitors, MTX, cyclosporine, CTLA4Ig and 
Janus kinase). Different drugs can be administered in sequential schedules (step-up, commencing with drugs with lower costs and toxicity; top-down, commencing with drugs with high potency to induce a remission that can be maintained using other drugs with higher safety and lower costs) or combination treatments, to exploit their different mechanisms of action (7-9).

However, the preclinical data regarding how these schedules act on the underlying mechanisms of the pathogenesis and progression of rheumatic diseases (such as activation and spreading of autoimmunity, while preserving protective immunity) is poor.

An in vitro model, in which the fate of unstimulated and stimulated cells could be differentially tracked by flow cytometry, was developed to reproduce an experimental condition relevant to the pathogenesis of rheumatic diseases, in which only certain lymphocytes are hypothesized to undergo antigen-driven activation. This model was used to investigate differences in action and possible synergisms between the two DMARDs, MTX and Tofa, by analysing the proliferation, activation and cytokine secretion of T cells. It was found that the stimulated lymphocytes and unstimulated cells were affected by the treatment. Furthermore, resting cells undergo a bystander activation due to the presence of stimulated lymphocytes and are consequently affected by the treatment.

\section{Materials and methods}

Experimental plan. The present study was approved by the Institutional Review Board of the Institute for Maternal and Child Health, IRCCS 'Burlo Garofolo', Trieste, Italy (RC 28/2009). Peripheral blood mononuclear cells (PBMCs) were obtained from the buffy coats of six healthy donors subsequent to receiving written consent. PBMCs were cultured for 9 days according to the experimental plan presented in Fig. 1. Briefly, at day 0, PBMCs were obtained by density gradient centrifugation (at $500 \mathrm{x}$ g for $30 \mathrm{~min}$ without breaking, at room temperature) and divided into two groups. The first group contained $6 \times 10^{7}$ cells stained with $5 \mu \mathrm{M}$ CellTrace ${ }^{\mathrm{TM}}$ Oregon Green ${ }^{\circledR} 488$ Carboxylic Acid Diacetate, Succinimidyl Ester (Carboxy-DFFDA, SE; CellTrace ${ }^{\mathrm{TM}}$ Green) obtained from Thermo Fisher Scientific, Inc. (Waltham, MA, USA) and stimulated with $2 \mu \mathrm{g} / \mathrm{ml}$ phytohaemagglutinin (PHA; Biochrom $\mathrm{GmbH}$, Berlin, Germany), while the second group $\left(4 \times 10^{7}\right.$ cells) was stained with $5 \mu \mathrm{M}$ CellTrace ${ }^{\mathrm{TM}}$ Violet Cell Proliferation kit (CellTrace ${ }^{\mathrm{TM}}$ Violet; Thermo Fisher Scientific, Inc.) and maintained in a culture without direct stimulation (non-stimulated; NS-cells). After $24 \mathrm{~h}$ of independent culture (day 1), the two groups of cells were washed with saline (Braun Melsungen AG, Melsungen, Germany) to remove the PHA and were re-plated in the presence of $200 \mu \mathrm{M}$ MTX (Teva Pharmaceuticals Industries, Ltd., Milan, Italy) or $10 \mu \mathrm{M}$ Tofa (Sigma-Aldrich, St Louis, MO, USA), or in the absence of drugs. Concentrations of Tofa and MTX were selected on the basis of previous studies $(10,11)$ to achieve an inhibitory effect on cell proliferation and activation without a marked effect on cell viability. To mimic a pathogenic setting containing stimulated and unstimulated cells, PHA-stimulated cells and NS-cells were plated together at a 1:4 ratio (designated as MIX). On day 5, the cells were washed to remove the first drug and were plated in the presence or absence of the second drug (either Tofa or MTX) for a further four days (day 9). For all experiments, cells were incubated at $37^{\circ} \mathrm{C}$ (atmosphere, $5 \% \mathrm{CO}_{2}$ ) at a concentration of $10^{6}$ cells $/ \mathrm{ml}$ in X-VIVO ${ }^{\mathrm{TM}} 15$ medium (Lonza Verviers Sprl, Verviers, Belgium) supplemented with 10\% Human AB Serum (Sigma-Aldrich), $100 \mathrm{U} / \mathrm{ml}$ penicillin/streptomycin and $200 \mathrm{mM}$ L-glutamine (EuroClone S.p.A., Milan, Italy).

Flow cytometric analysis of proliferation and activation. At day 5 and day $9,2 \times 10^{5}$ cells were stained with allophycocyanine/Cy7-conjugated anti-human CD25 monoclonal antibody (final dilution, $2.5 \mu \mathrm{g} / \mathrm{ml}$; cat. no. 302614; BioLegend, Inc., San Diego, CA, USA) to evaluate activation. Simultaneously, staining with CellTrace ${ }^{\mathrm{TM}}$ was performed before cells were placed in the culture, to evaluate cell proliferation, which was assessed using a dye dilution assay following each cell division (12) and then expressed as a percentage of proliferated cells. Activation of cells was evaluated by measuring the median fluorescence intensity (MFI) of CD25. Data were obtained using a CyAn ${ }^{\mathrm{TM}}$ ADP cytometer (Beckman Coulter, Inc., Brea, CA, USA) and analysed with FlowJo software version 7.6 (Tree Star, Inc., Ashland, OR, USA).

Analysis of cytokines in culture supernatants. To measure the levels of cytokines released by the cells, at day 1,5 and 9, culture supernatants were aspirated, collected in a vial and stored at $-20^{\circ} \mathrm{C}$ until analysis. Concentrations were evaluated using the magnetic-beads suspension assay using Bio-Plex ${ }^{\circledR}$ technology (Bio-Plex Pro ${ }^{\mathrm{TM}}$, Human Cytokine 8-Plex Panel; Bio-Rad Laboratories, Inc., Hercules, CA, USA). The analysis included eight cytokines and chemokines as follows: IL-2, -4, -6, -8, -10, granulocyte-macrophage colony-stimulating factor, interferon- $\gamma$ (IFN- $\gamma$ ), TNF- $\alpha$. Multiplex assay suspension data were acquired using a Bio-Plex ${ }^{\circledR} 200$ reader (Bio-Rad Laboratories, Inc.), and data output was analysed using a high-speed digital processor (Bio-Plex Multiplex System; Bio-Rad Laboratories, Inc.). Concentrations are presented in units $(\mathrm{pg} / \mathrm{ml})$.

Statistical analysis. All results are presented as means of two independent experiments \pm standard deviation. Statistical significance was calculated using a one-way analysis of variance and Tukey's multiple comparison post-test. Analysis was performed using GraphPad Prism software (version 5.0; GraphPad Software, Inc., La Jolla, CA, USA). $\mathrm{P}<0.05$ was considered to indicate a statistically significant difference.

\section{Results}

When administered as the first drug, MTX blocked proliferation, but Tofa did not exert significant effects. After the first $24 \mathrm{~h}$ of culture in the presence or absence of PHA, cells were washed, treated with the first drug, MTX, and incubated for a further 4 days. Cells were then washed and incubated in the presence of the second drug, Tofa, for a further 4 days. At the end of the incubation periods (days 5 and 9), the cells were analysed by flow cytometry to assess the activation and proliferation. At day 5 in the MIX condition, the proliferation of PHA-stimulated cells was strongly reduced by MTX treatment: $\mathrm{PHA}_{\mathrm{MIX}}, 83.35 \pm 4.65$ vs. $\mathrm{PHA}_{\mathrm{MIX}}+\mathrm{MTX}, 13.39 \pm 9.72 \%$ $(\mathrm{P}<0.0001$; Fig. 2A). In addition, NS-cells in the MIX 


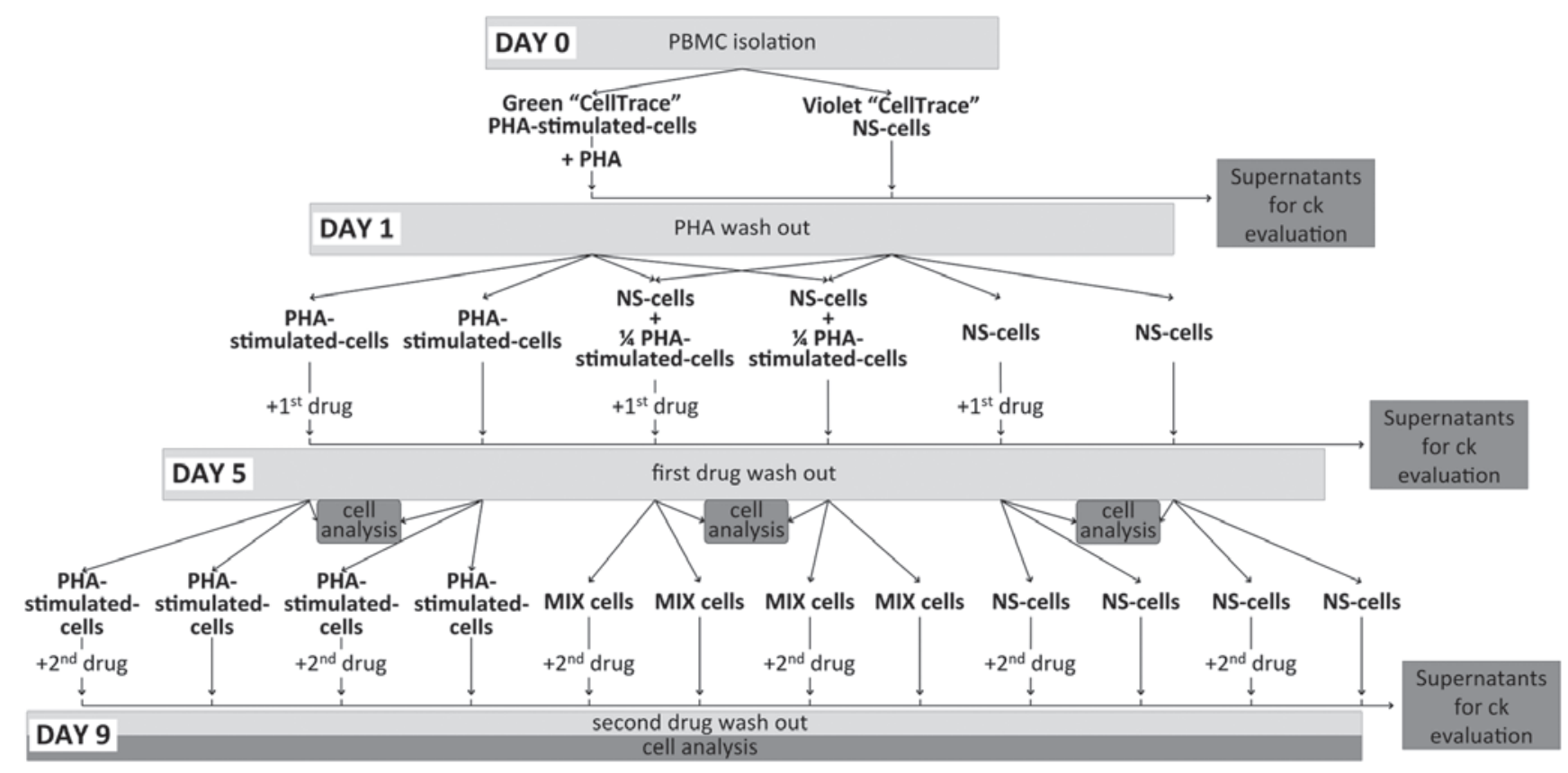

Figure 1. Schematic of the experimental plan. The plan depicts the culture time line and the sequential treatment with the two drugs. The time-point of analysis is also indicated. NS, NS cells tracked with CellTrace ${ }^{\mathrm{TM}}$ Violet; PHA, PHA-stimulated cells tracked with CellTrace ${ }^{\mathrm{TM}}$ Green; MIX cells, co-culture of PHA-stimulated cells and NS-cells (ratio, 1:4). NS, non-stimulated; ck, cell cytokine; PBMC, peripheral blood mononuclear cell; PHA, phytohaemagglutinin.

condition demonstrated low percentages of proliferation that appeared to be marginally affected by MTX, although the difference was not statistically significant: $\mathrm{NS}_{\mathrm{MIX}}$, $19.25 \pm 3.55$ vs. $\mathrm{NS}_{\mathrm{MIX}}+\mathrm{MTX}, 8.85 \pm 3.65 \%$ (Fig. 2A). Following the washout, at day 9 , the antiproliferative effect of MTX on PHA-stimulated cells in the MIX condition continued to be evident: $\mathrm{PHA}_{\mathrm{MIX}}, 86.40 \pm 7.90$ vs. $\mathrm{PHA}_{\mathrm{MIX}}+\mathrm{MTX}$, $22.65 \pm 5.15 \%(\mathrm{P}<0.0001)$.

The administration of Tofa on day 5 did not demonstrate any further antiproliferative effect on PHA-stimulated cells regardless of a previous treatment with MTX: PHA $\mathrm{MIX}_{\text {, }}$ $86.40 \pm 7.90$ vs. PHA $_{\text {MIX }}$ + Tofa, $87.15 \pm 7.75 \%$; PHA $_{\text {MIX }}+$ MTX, $22.65 \pm 5.15$ vs. $\mathrm{PHA}_{\mathrm{MIX}}+$ MTX / Tofa, $26.40 \pm 3.50 \%$ (Fig. 2B).

NS-cells in the MIX condition at day 9 showed marginal proliferation $\left(\mathrm{NS}_{\mathrm{MIX}}, 31.70 \pm 0.10 \%\right)$, which appeared to be reduced by the addition of MTX $\left(\mathrm{NS}_{\mathrm{MIX}}+\mathrm{MTX}, 12.50 \pm 0.80 \%\right)$ during the first time frame (days 1-5), although the difference was not statistically significant, and Tofa treatment alone during the second time frame (days 5-9) did not exert any effects $\left(\mathrm{NS}_{\mathrm{MIX}}+\mathrm{MTX}-\mathrm{Tofa}, 31.20 \pm 1.60 \%\right)$. Furthermore, the addition of Tofa to MTX-treated cells did not reveal any increase in the antiproliferative effect when compared with MTX alone: $\mathrm{NS}_{\mathrm{MIX}}+\mathrm{MTX}, 12.50 \pm 0.80$ vs. $\mathrm{NS}_{\mathrm{MIX}}+$ MTX-Tofa $15.25 \pm 1.55 \%$ (Fig. 2B).

Activation was not completely blocked by MTX. MTX induced a significant decrease in the level of CD25 expression in PHA-stimulated cells in the MIX condition: $\mathrm{PHA}_{\mathrm{MIX}}, 18.35 \pm 2.45$ vs. $\mathrm{PHA}_{\mathrm{MIX}}+\mathrm{MTX}, 9.03 \pm 1.27$ $(\mathrm{P}<0.05$; Fig. 2C). Whereas no differences in activation was evident in the NS-cells irrespective of the presence of MTX: $\mathrm{NS}_{\text {MIX }}, 7.86 \pm 0.07 ; \mathrm{NS}_{\mathrm{MIX}}+\mathrm{MTX}, 6.55 \pm 0.01$ (Fig. 2C). At day 9, there was a residual level of CD25 expression that was not influenced by any of the pharmacologic treatments: $\mathrm{PHA}_{\mathrm{MIX}}, 8.75 \pm 0.05 ; \mathrm{PHA}_{\mathrm{MIX}}+\mathrm{MTX}, 7.30 \pm 0.66$; $\mathrm{PHA}_{\mathrm{MIX}}+$ MTX-Tofa, 7.44 $\pm 0.52 ; \mathrm{PHA}_{\mathrm{MIX}}+$ Tofa, $8.56 \pm 0.64$ (Fig. 2D).

Following withdrawal of MTX treatment, cells underwent a cytokine rebound, which was partially restored by Tofa. At day 5, following PHA stimulation, the cells exhibited higher concentrations of all of the cytokines when compared with the NS condition. In addition, MTX treatment appeared to reduce the cytokine secretion in PHA-stimulated cells alone and in the MIX condition, but to a reduced extent. An exception was observed for the concentration of IL-2, which was marginally increased after MTX treatment (Fig. 3).

By contrast, at day 9, PHA-stimulated cells that had initially undergone MTX treatment showed increased production of all cytokines when compared with the untreated cells.

Treatment with Tofa subsequent to MTX led to a reduced cytokine secretion when compared with MTX treatment alone, although the concentrations remained higher than those of the untreated PHA-stimulated cells. In the MIX condition, the effects of the drug treatments demonstrated similar trends to those of PHA stimulation, although the concentrations were generally lower.

Furthermore, higher concentrations of IL-2 were demonstrated in PHA-stimulated cells treated with MTX + Tofa, when compared with other conditions (Fig. 3). Notably, no significant difference in IL-8 concentration was demonstrated regardless of the stimulus and of the drug treatment at day 1 and 5; conversely, a higher secretion was observed at day 9 in the culture supernatants of the MIX-condition, when cells were treated with Tofa alone or in combination with MTX (Fig. 3).

Tofa demonstrated a low antiproliferative effect on PHA-stimulated cells, increased by the addition of MTX. Cells 
A

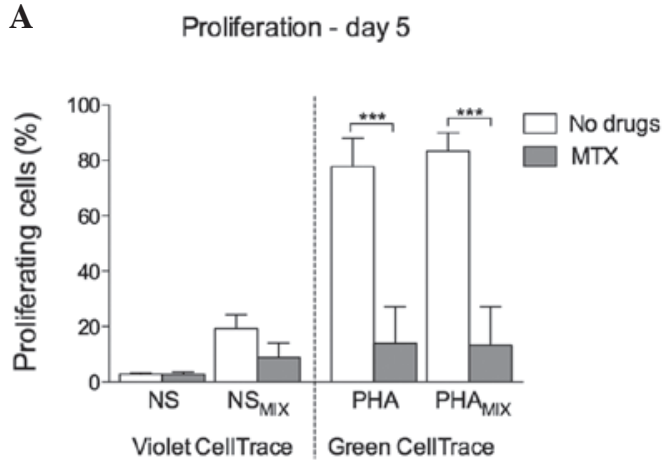

C

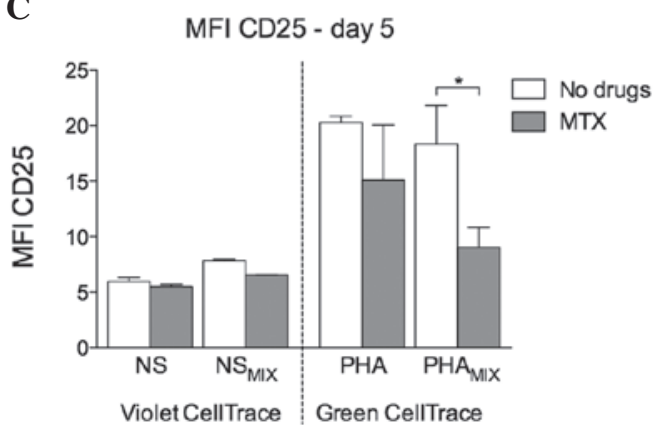

B

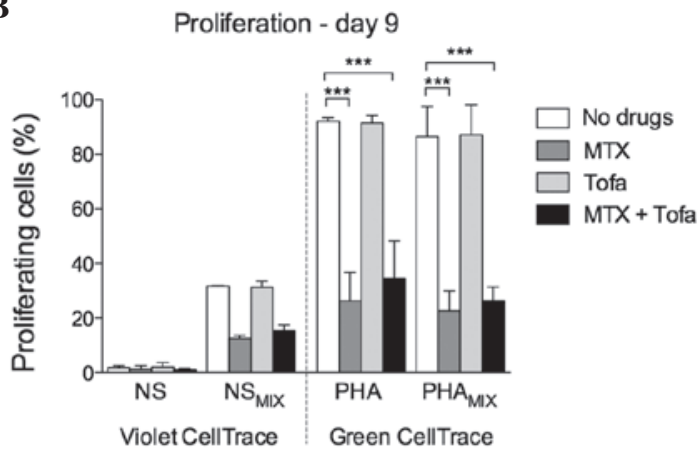

D

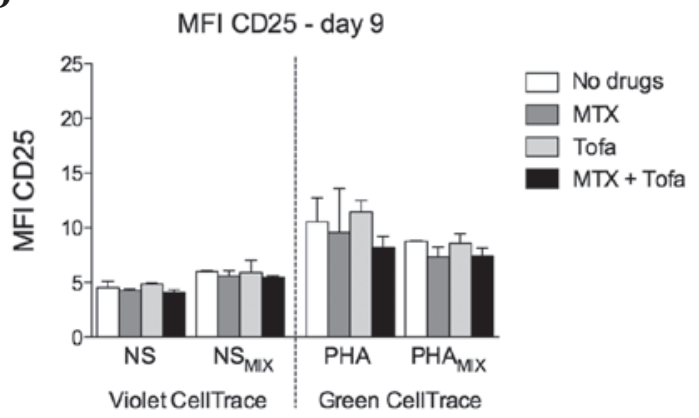

Figure 2. Cell proliferation and activation following treatment with MTX then Tofa. Percentage of proliferating cells after (A) 5 and (B) 9 days of incubation with MTX then Tofa. Activation evaluated as MFI of CD25 at (C) day 5 and (D) day 9 of drug treatments. NS, NS-cells cultured alone; PHA, PHA-stimulated cells cultured alone; $\mathrm{NS}_{\mathrm{MIX}}$, NS-cells co-cultured in the presence of PHA-stimulated cells; $\mathrm{PHA}_{\mathrm{MIX}}$, PHA-stimulated cells co-cultured in the presence of NS-cells. To distinguish between the two populations, NS-cells were stained with CellTrace ${ }^{\mathrm{TM}}$ Violet, while PHA-stimulated cells were stained with CellTrace ${ }^{\mathrm{TM}}$ Green. ${ }^{*} \mathrm{P}<0.05$ and ${ }^{* * *} \mathrm{P}<0.0001$. NS, non-stimulated; MTX, methotrexate; PHA, phytohaemagglutinin; Tofa, tofacitinib; MFI, median fluorescence intensity.

were cultured for $24 \mathrm{~h}$ with or without PHA, and then washed and plated in the presence or absence of Tofa. After 4 days of culture (day 5), cells were washed and incubated in the presence or absence of MTX for a further 4 days (day 9). When Tofa was administered as the first drug in the MIX condition, proliferation of PHA-stimulated cells at day 5 tended to be marginally decreased: $\mathrm{PHA}_{\mathrm{MIX}}, 73.60 \pm 21.40 \mathrm{vs}$. $\mathrm{PHA}_{\mathrm{MIX}}+$ Tofa, $55.00 \pm 25.60 \%$. NS-cells in the MIX condition displayed an appreciable proliferation rate, which was partly reduced by Tofa treatment: $\mathrm{NS}_{\mathrm{MIX}}, 15.45 \pm 4.15$ vs. $\mathrm{NS}_{\mathrm{MIX}}+$ Tofa, $8.15 \pm 4.26 \%$ (Fig. 4A).

At day 9, sequential treatment with Tofa and MTX induced an additional reduction of proliferation when compared with Tofa treatment alone: $\mathrm{PHA}_{\mathrm{MIX}}+$ Tofa-MTX, $38.41 \pm 37.09$ vs. PHA $_{\text {MIX }}+$ Tofa, $55.65 \pm 23.65 \%$. Furthermore, NS-cells displayed low proliferation: $\mathrm{NS}_{\mathrm{MIX}}, 24.65 \pm 10.45 \%$, which was marginally influenced by treatment with Tofa: $\mathrm{NS}_{\mathrm{MIX}}+$ Tofa, $13.31 \pm 11.20 \%$, and was not affected by the addition of MTX during the second incubation: $\mathrm{NS}_{\mathrm{MIX}}+\mathrm{MTX}$, $25.75 \pm 13.95 \%$ NS $_{\mathrm{MIX}}$ + Tofa-MTX, 15.33 $\pm 11.27 \%$ (Fig. 4B).

Tofa induced an enduring reduction in activation, but with reduced effect on NS-cells and MTX exerted little effect. At day 5, although the differences observed were not significant, Tofa treatment appeared to decrease the activation of PHA-stimulated cells in the MIX condition: PHA $_{\text {MIX }}, 18.30 \pm 8.30$ vs. PHA $_{\text {MIX }}+$ Tofa, 7.04 \pm 1.40 (Fig. 4C). Accordingly, NS-cells underwent marginal activation $\left(\mathrm{NS}_{\mathrm{MIX}}, 6.95 \pm 3.16\right)$ that was reduced by Tofa $\left(\mathrm{NS}_{\mathrm{MIX}}+\right.$ Tofa, $3.89 \pm 0.32$; Fig. 4C).
Conversely, at day 9, the addition of MTX as the second drug did not appear to have an effect on PHA-stimulated cells, regardless of the previous Tofa treatment: $\mathrm{PHA}_{\mathrm{MIX}}, 10.53 \pm 2.17$; $\mathrm{PHA}_{\text {MIX }}+$ Tofa, 7.74 \pm 0.22 PHA $_{\text {MIX }}+$ MTX, 11.04 \pm 2.16 ; PHA $_{\text {MIX }}+$ Tofa-MTX, 7.04 \pm 0.36 (Fig. 4D).

Tofa treatment reduced IFN- $\gamma$ and IL-4 production, but MTX did not exert an effect. At day 5, Tofa treatment reduced cytokine secretion, particularly secretion of IFN- $\gamma$ : PHA, 21,857. $07 \pm 1,701.98$ vs. PHA + Tofa, 1,978.82 $\pm 120.12 \mathrm{pg} / \mathrm{ml}(\mathrm{P}<0.05)$ and of IL-4: PHA, 6.74 \pm 0.91 vs. PHA + Tofa, $4.42 \pm 0.44 \mathrm{pg} / \mathrm{ml}$ $(\mathrm{P}<0.05)$ in PHA-stimulated cells and this trend was maintained in the MIX condition (Fig. 5).

At day 9, MTX alone did not induce a reduction of cytokine secretion in the PHA-stimulated cells when compared with untreated cells, although MTX treatment exerted an additional effect to the Tofa treatment, in particular for IL-4 and IFN- $\gamma$. In the MIX condition, this effect was less evident.

Drug treatments, in PHA-stimulated cells, induced a higher production of IL-2. By contrast, Tofa treatment, alone and in combination with MTX, induced a decreased production of IL-8 (Fig. 5).

\section{Discussion}

The therapeutic approach in rheumatic diseases is based upon the administration of anti-inflammatory glucocorticoids, DMARDs or biologics. Recently, novel drugs are also being developed (3), and there is evidence to suggest that combined or sequential administration of well-established therapeutic 

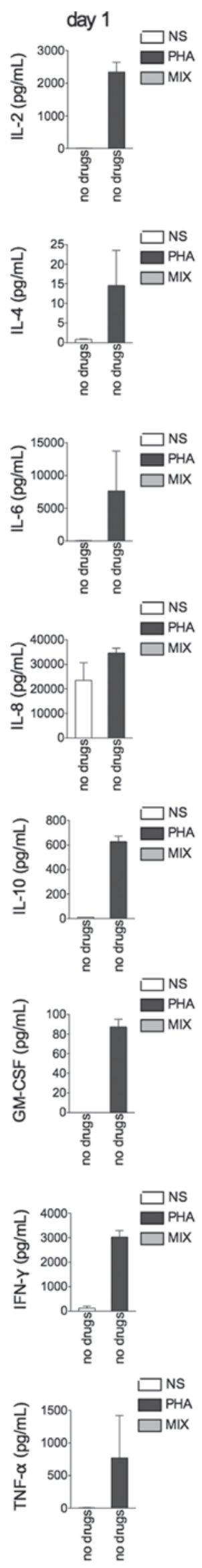
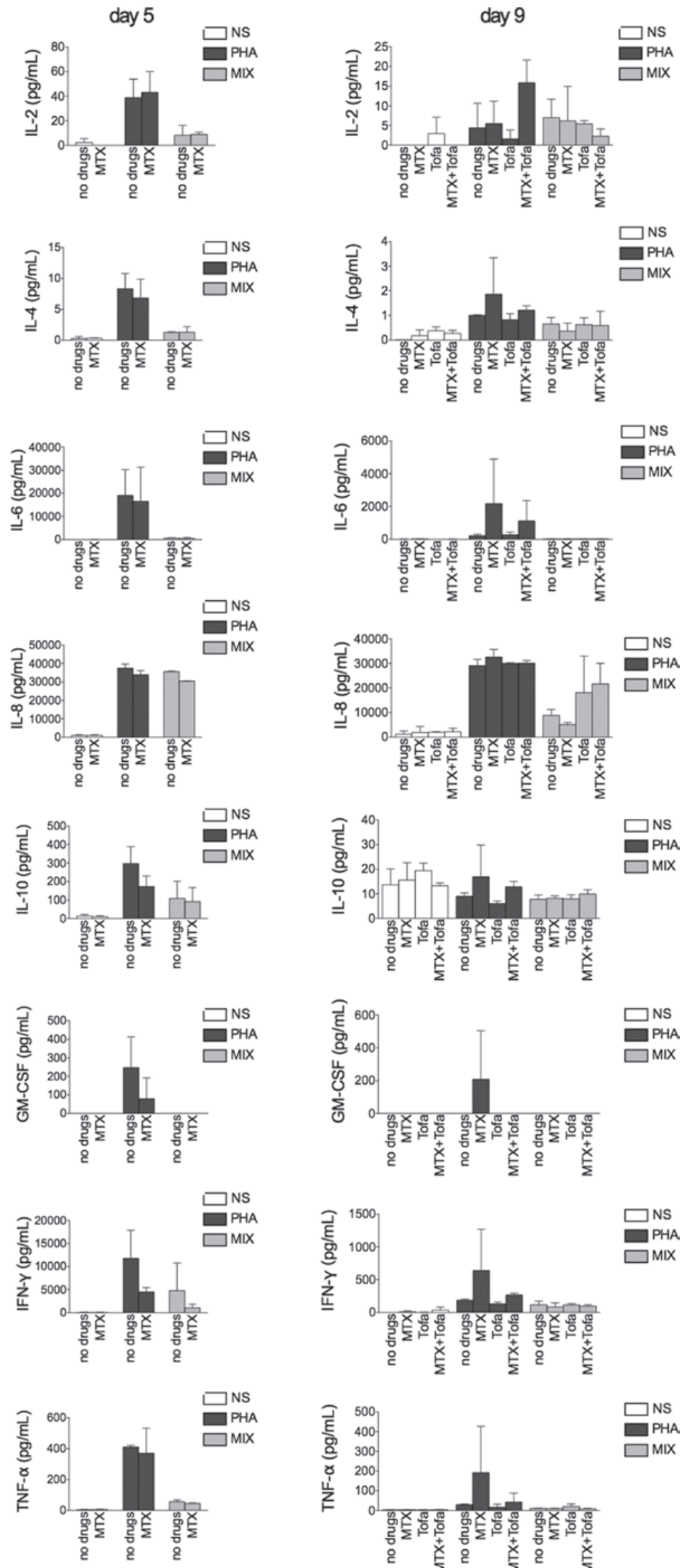

Figure 3. Cytokine release in supernatants after sequential treatment with MTX and Tofa. Bar charts represent the cytokine concentration, expressed as pg/ml, evaluated with Bio-Plex ${ }^{\circledR}$ technology. NS, supernatants from unstimulated cells; PHA, supernatants from PHA-stimulated cells; MIX, supernatants from a co-culture of NS and PHA-stimulated cells. NS, non-stimulated; IL, interleukin; GM-CSF, granulocyte-macrophage colony-stimulating factor; IFN- $\gamma$, interferon- $\gamma$; TNF- $\alpha$, tumour necrosis factor- $\alpha$; PHA, phytohaemagglutinin; MTX, methotrexate; Tofa, tofacitinib. 
A

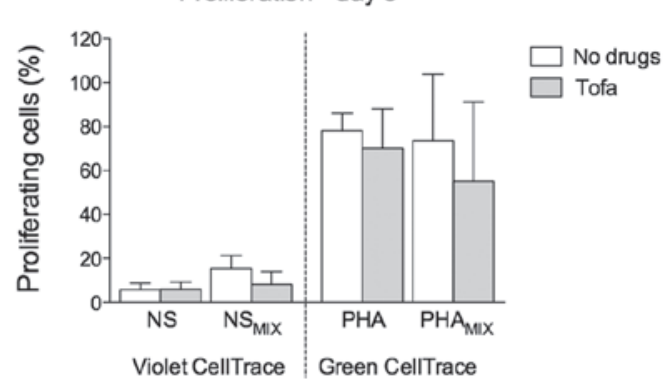

C

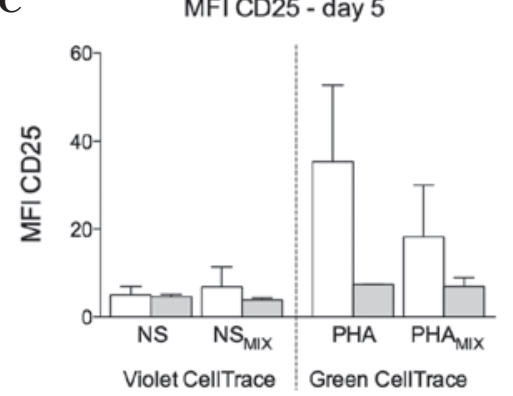

B

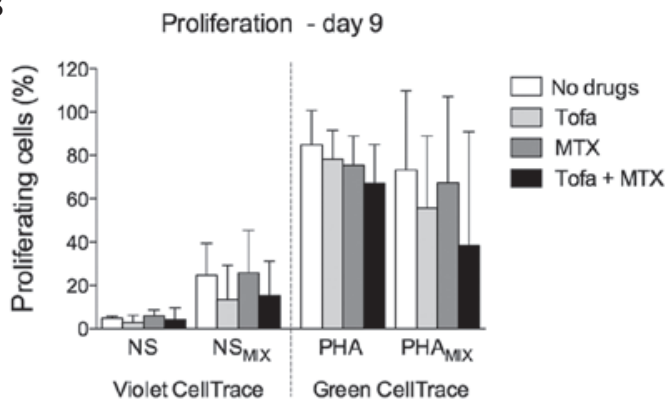

D

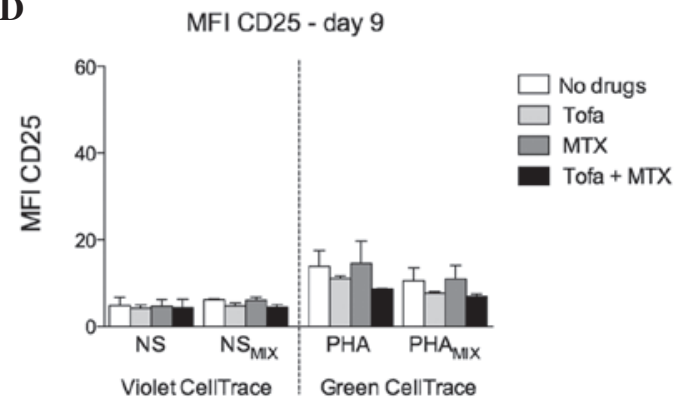

Figure 4. Effect of sequential treatment with Tofa and MTX on cell proliferation and activation. Percentage of proliferating cells (A) after the incubation in presence of Tofa (day 5) and (B) following subsequent incubation with MTX at day 9. Cell activation, presented as MFI, of CD25 (C) at day 5 and (D) at

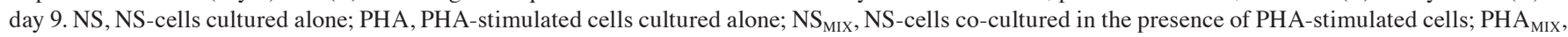
PHA-stimulated cells co-cultured in the presence of NS-cells. To distinguish between the two populations, NS-cells were stained with CellTrace ${ }^{\mathrm{TM}}$ Violet, while the PHA-stimulated cells were stained with CellTrace ${ }^{\mathrm{TM}}$ Green. NS, non-stimulated; PHA, phytohaemagglutinin; MTX, methotrexate; Tofa, tofacitinib; MFI, median fluorescence intensity.

strategies improves the patient outcome without the high costs of biologics $(7,13)$.

The possible mechanisms of action of combination therapy have been poorly investigated in the pre-clinical and laboratory settings. However, there is the potential to obtain greater advantages in terms of better outcomes for patients, higher safety and lower costs by exploiting the different characteristics of novel and existing drugs in combination treatments.

Among the drugs available for rheumatic diseases, MTX has achieved marked results in past decades and Tofa is among the most innovative, recently developed molecules.

Although there are clinical studies that compare the two drugs (14) or that evaluate their use in combined treatments (15), to the best of our knowledge, data regarding in vitro models, which examine the mechanisms of action $(10,11,16)$ to optimize their pharmacological action when used in combination, are lacking.

The present study demonstrated that MTX and Tofa exerted different effects on cell activation and proliferation, and it was proposed that these differences may be exploited by sequential therapeutic strategies, to maximize the anti-rheumatic effect. MTX showed a persistent antiproliferative effect and a moderate downregulation of activation on PHA-stimulated cells, which was not influenced by subsequent Tofa treatment. By contrast, Tofa marginally effected activation, but only slight antiproliferative action that, however, could be potentiated by sequential treatment with MTX.

Recent studies have demonstrated that Tofa only exerts its effect on recently activated cells. Doses higher than those typically administered block the activation and proliferation of stimulated lymphocytes, but the effect is transient, with the levels of activation and proliferation restored following withdrawal of the drug (10). The present study proposed that proliferation of the cells following Tofa treatment-and-withdrawal may be exploited to render cells increasingly sensitive to sequential treatment with other drugs, such as MTX. Indeed, MTX demonstrated a strong antiproliferative effect on recently activated cells, during the first incubation, although not on continuously proliferating cells during the second time frame. However, the antiproliferative effect of MTX during the second time frame was evident when Tofa was administered first, directly after cell stimulation.

Furthermore, on day 5, the two drugs tended to reduce the secretion of pro-inflammatory cytokines by cells recently stimulated with PHA; notably, IFN- $\gamma$ and IL-4 levels were significantly reduced in Tofa-treated cells $(\mathrm{P}<0.05)$ and this trend was also observed in MTX-treated cells, although this difference was not identified to be statistically significant.

By contrast, during the second time frame, the use of different sequences of drugs caused conflicting effects on cytokine secretion. When the sequence of MTX + Tofa was used, an increased secretion of cytokines by cells treated with MTX only during the first time frame was observed at day 9, as compared with the other treatments. This effect may indicate that cell activation became unhindered following withdrawal of MTX, which resulted in a revived level of activation; conversely, when Tofa was added to the culture, a reduction of cytokine secretion was observed, when Tofa was administered alone or following MTX.

When MTX was administered alone during the second time frame, it did not reduce, but caused an increase in, cytokine release; however, treatment with Tofa in the first incubation period resulted in a greater inhibition of cytokine release, which 
day 1
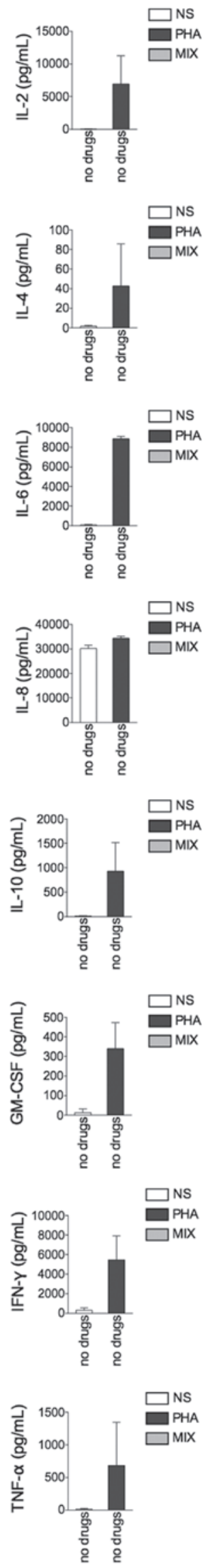

day 5
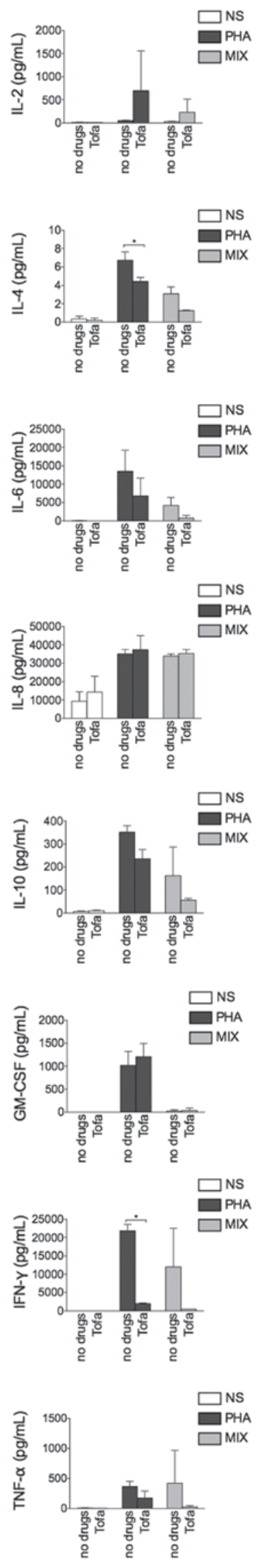

day 9
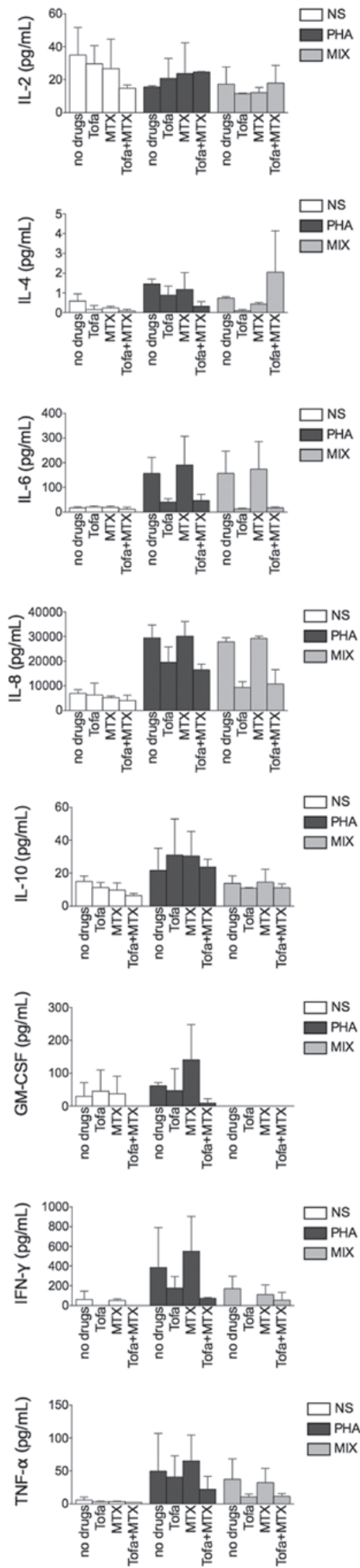

Figure 5. Cytokine evaluation in cell culture supernatants following treatment with Tofa then MTX. The bar charts represent the cytokine concentration, expressed as pg/ml, evaluated with Bio-Plex ${ }^{\circledR}$ technology. NS, supernatants from unstimulated cells; PHA, supernatants from PHA-stimulated cells; MIX, supernatants from mixed culture of NS and PHA-stimulated cells. * $\mathrm{P}<0.05$. NS, non-stimulated; IL, interleukin; GM-CSF, granulocyte-macrophage colony-stimulating factor; IFN- $\gamma$, interferon- $\gamma$; TNF- $\alpha$, tumour necrosis factor- $\alpha$; PHA, phytohaemagglutinin; MTX, methotrexate; Tofa, tofacitinib. 
was more marked following Tofa + MTX treatment. This was likely due to the inability of the second drug alone to act on the cells that were activated later, and could suggest that sequential drug treatments may themselves be influenced by timing and sequence.

Exceptions were observed for IL-8 and -2. IL-8 showed high levels of secretion at all times and did not undergo any significant variation regardless of the treatment; this may be due to the fact that this cytokine was predominantly produced by other cells, rather than $\mathrm{T}$ lymphocytes, which did not undergo drug treatments. Conversely, IL-2 concentration increased following Tofa treatment, whether Tofa was administered as the first drug or following MTX treatment. This may be due to positive feedback from IL-2 signalling, when the signalling pathway was blocked by Tofa. Notably, in the MIX condition, the trend of the effects on cytokine secretion, observed in PHA-stimulated cells, was largely reproduced at day 5 . During the second time frame, certain differences between PHA-stimulated cells and the MIX condition may have been apparent, as the supernatant was collected from the culture wells and it represents the cytokines that were produced by the total of the cells; whereas, in the MIX condition, only a quarter of the cells were pre-stimulated with PHA. It should be noted, however, that the present study had certain limitations: Due to the experimental design, a large amount of cells was required; therefore, buffy coats from healthy donors were used, which showed a higher variability in results than peripheral blood cells or cell lines. Moreover, the standard deviation at day 9 was very large, which was probably due to the long culture period.

Furthermore, the present study proposed an in vitro model that was constructed to simulate rheumatic disease, where only certain lymphocytes are activated (putative pathogenic lymphocytes) while the majority remain unstimulated (17). In this model, the unstimulated cells (NS-cells) were stained with CellTrace ${ }^{\mathrm{TM}}$ Violet, while PHA-stimulated cells were stained with CellTrace ${ }^{\mathrm{TM}}$ Green and the two populations were co-cultured. The staining with different tracers allowed independent tracking of the fate of the directly stimulated and unstimulated populations. The activation and proliferation of NS-cells in the MIX condition was notable. These cells tended to proliferate, which may have been due to bystander stimulation. These cells were sensitive to the two drugs administered during the first time frame, while drug treatment in the second time frame, sequential or not, appeared to exert no effect; which was consistent with the result of cytokine secretion in the supernatant.

This particular issue must be considered further. In vivo, certain lymphocytes in the same milieu of pathogenic cells may undergo bystander activation, possibly contributing to the spread of autoimmune activation and disease progression. Indeed, $T$ cells that are stimulated by a cytokine cocktail induce the release of proinflammatory cytokines from monocytes. A previous study identified that signalling pathways in the responding monocytes were identical, regardless of whether the monocytes were placed in contact with $\mathrm{T}$ cells isolated from rheumatoid arthritis synovial tissue (18).

A previous study indicated that bystander cells accumulate pathogenic potential with age (19), therefore, it is of particular interest to control these cells, particularly in long-term treatment strategies. Furthermore, therapy tends to modify the course of rheumatic disease, possibly indicating a role of selection due to pathogenic cell clones escaping from drug action; although, to the best of our knowledge, the underlying mechanisms remain to be fully demonstrated, it is likely that the escaping clones are the most pathogenic ones and are able to spread the immune defect $(20,21)$. Thus, these considerations support the current therapeutic strategies, which are based on different approaches that combine drugs in consecutive use (such as step-up and top-down).

In conclusion, the present study does not directly correlate with the mechanisms of rheumatic disease in vivo; however, does indicate that it is possible to obtain a simplified in vitro model to evaluate the effect of sequential treatment methods with various anti-rheumatic drugs. Furthermore, this model allows the outcome of the primary activated cells (i.e pathogenic lymphocytes) to be tracked, but also enables evaluation of the fate of the cells that are bystander-activated by the immunological milieu during active disease.

\section{Acknowledgements}

The present study was supported by a grant from the Maternal and Child Institute 'Burlo Garofolo’ (grant no. RC 28/2009).

\section{References}

1. McGonagle D, Aziz A, Dickie LJ and McDermott MF: An integrated classification of pediatric inflammatory diseases, based on the concepts of autoinflammation and the immunological disease continuum. Pediatr Res 65: 38R-45R, 2009.

2. Uhlig T, Moe RH and Kvien TK: The burden of disease in rheumatoid arthritis. Pharmacoeconomics 32: 841-851, 2014.

3. Kremer JM, Bloom BJ, Breedveld FC, Coombs JH, Fletcher MP, Gruben D, Krishnaswami S, Burgos-Vargas R, Wilkinson B, Zerbini CA and Zwillich SH: The safety and efficacy of a JAK inhibitor in patients with active rheumatoid arthritis: Results of a double-blind, placebo-controlled phase IIa trial of three dosage levels of CP-690,550 versus placebo. Arthritis Rheum 60: 1895-1905, 2009.

4. Tanaka Y and Yamaoka K: JAK inhibitor tofacitinib for treating rheumatoid arthritis: From basic to clinical. Mod Rheumatol 23: 415-424, 2013.

5. Shi JG, Chen X, Lee F, Emm T, Scherle PA, Lo Y, Punwani N, Williams WV and Yeleswaram S: The pharmacokinetics, pharmacodynamics, and safety of baricitinib, an oral JAK 1/2 inhibitor, in healthy volunteers. J Clin Pharmacol 54: 1354-1361, 2014.

6. Keystone EC, Taylor PC, Drescher E, Schlichting DE, Beattie SD, Berclaz PY, Lee CH, Fidelus-Gort RK, Luchi ME, Rooney TP et al: Safety and efficacy of baricitinib at 24 weeks in patients with rheumatoid arthritis who have had an inadequate response to methotrexate. Ann Rheum Dis 74: 333-340, 2015.

7. Wailoo A, Hernández MA, Scott IC, Ibrahim F and Scott DL: Cost-effectiveness of treatment strategies using combination disease-modifying anti-rheumatic drugs and glucocorticoids in early rheumatoid arthritis. Rheumatology (Oxford) 53: 1773-1777, 2014.

8. Verschueren P, Esselens G and Westhovens R: Daily practice effectiveness of a step-down treatment in comparison with a tight step-up for early rheumatoid arthritis. Rheumatology (Oxford) 47: 59-64, 2008.

9. Lee YH, Bae SC and Song GG: Comparative efficacy and safety of tofacitinib, with or without methotrexate, in patients with active rheumatoid arthritis: A Bayesian network meta-analysis of randomized controlled trials. Rheumatol Int 35: 1965-1974, 2015.

10. Piscianz E, Valencic E, Cuzzoni E, De Iudicibus S, De Lorenzo E, Decorti $G$ and Tommasini A: Fate of lymphocytes after withdrawal of tofacitinib treatment. PLoS One 9: e85463, 2014.

11. Piscianz E, Cuzzoni E, De Iudicibus S, Valencic E, Decorti G and Tommasini A: Differential action of 3-hydroxyanthranilic acid on viability and activation of stimulated lymphocytes. Int Immunopharmacol 11: 2242-2245, 2011. 
12. Lyons AB, Blake SJ and Doherty KV: Flow cytometric analysis of cell division by dilution of CFSE and related dyes. Curr Protoc Cytom Chapter 9: Unit9.11, 2013.

13. O'Dell JR, Mikuls TR, Taylor TH, et al; CSP 551 RACAT Investigators: Therapies for active rheumatoid arthritis after methotrexate failure. N Engl J Med 369: 307-318, 2013.

14. Lee EB, Fleischmann R, Hall S, Wilkinson B, Bradley JD, Gruben D, Koncz T, Krishnaswami S, Wallenstein GV, Zang C, et al: Tofacitinib versus methotrexate in rheumatoid arthritis. N Engl J Med 370: 2377-2386, 2014.

15. Burmester GR, Blanco R, Charles-Schoeman C, Wollenhaupt J, Zerbini C, Benda B, Gruben D, Wallenstein G, Krishnaswami S, Zwillich SH, et al: Tofacitinib (CP-690,550) in combination with methotrexate in patients with active rheumatoid arthritis with an inadequate response to tumour necrosis factor inhibitors: A randomised phase 3 trial. Lancet 381: 451-460, 2013.

16. Maeshima K, Yamaoka K, Kubo S, Nakano K, Iwata S, Saito K, Ohishi M, Miyahara H, Tanaka S, Ishii K, et al: The JAK inhibitor tofacitinib regulates synovitis through inhibition of interferon- $\gamma$ and interleukin-17 production by human CD4+ T cells. Arthritis Rheum 64: 1790-1798, 2012.
17. Cope AP, Schulze-Koops $\mathrm{H}$ and Aringer M: The central role of $\mathrm{T}$ cells in rheumatoid arthritis. Clin Exp Rheumatol 25 (5 Suppl 46): S4-S11, 2007.

18. Brennan FM, Smith NM, Owen S, Li C, Amjadi P, Green P, Andersson A, Palfreeman AC, Hillyer P, Foey A, et al: Resting CD4+effector memory T cells are precursors of bystander-activated effectors: A surrogate model of rheumatoid arthritis synovial T-cell function. Arthritis Res Ther 10: R36, 2008.

19. Kobayashi M, Yasui N, Ishimaru N, Arakaki R and Hayashi Y: Development of autoimmune arthritis with aging via bystander $\mathrm{T}$ cell activation in the mouse model of Sjögren's syndrome. Arthritis Rheum 50: 3974-3984, 2004.

20. Kobari Y, Misaki Y, Setoguchi K, Zhao W, Komagata Y, Kawahata K, Iwakura Y and Yamamoto K: T cells accumulating in the inflamed joints of a spontaneous murine model of rheumatoid arthritis become restricted to common clonotypes during disease progression. Int Immunol 16: 131-138, 2004.

21. VanderBorght A, Geusens P, Raus J and Stinissen P: The autoimmune pathogenesis of rheumatoid arthritis: Role of autoreactive $\mathrm{T}$ cells and new immunotherapies. Semin Arthritis Rheum 31: 160-175, 2001. 